\title{
Regulation of adiponectin receptors in hepatocytes by the peroxisome proliferator-activated receptor-y agonist rosiglitazone
}

Received: 5 January 2006 / Accepted: 27 January 2006 / Published online: 12 April 2006

C) Springer-Verlag 2006

\begin{abstract}
Aims/hypothesis: Adiponectin is an adipocytederived hormone that plays a critical role in the development of type 2 diabetes via interaction with adiponectin receptors 1 (ADIPOR1) and 2 (ADIPOR2). Rosiglitazone is a peroxisome proliferator-activated receptor- $\gamma$ (PPARG) agonist that is widely used in the treatment of type 2 diabetes. We hypothesised that rosiglitazone regulates lipid and glucose metabolism through modulation of the expression of adiponectin receptors in the liver. Methods: The expression of ADIPOR1 and ADIPOR2 was analysed in HepG2 hepatocytes. The promoters of adiponectin receptors were isolated and used to analyse the transcriptional regulation. The expression of adiponectin receptors in the liver was determined in mice treated with rosiglitazone. Results: Rosiglitazone elevated the mRNA and protein levels of ADIPOR2 and stimulated ADIPOR2 promoter in HepG2 cells. Analysis with the ADIPOR2 promoter revealed a putative rosiglitazone-responsive region that contained a glucocorticoid receptor (GR)binding element. The GR agonist dexamethasone synergised with rosiglitazone to stimulate the ADIPOR2 promoter wheras the GR antagonist RU486 abolished this stimulation. Treatment of mice with rosiglitazone elevated the expression of ADIPOR2 in the liver. Conclusions/interpretation: This study indicates that rosiglitazone can elevate the expression of ADIPOR2 in hepatocytes. Our data also suggest that the PPARG agonist rosiglitazone can interact functionally with a GR element in the ADIPOR2 promoter to mediate stimulation of transcription. This study thus reveals a new paradigm underlying the therapeutic effect of PPARG activators in the treatment of type 2 diabetes.
\end{abstract}

\footnotetext{
X. Sun $\cdot$ R. Han $\cdot$ Z. Wang $\cdot$ Y. Chen $(\bowtie)$

Institute for Nutritional Sciences, Shanghai Institute for

Biological Sciences (SIBS), Chinese Academy of Sciences,

294 Tai Yuan Rd., Shanghai, 200031, People's Republic of China

e-mail: ychen3@sibs.ac.cn

Tel.: +86-21-54920916

Fax: +86-21-54920291
}

Keywords Adiponectin - ADIPOR1 · ADIPOR2 . Diabetes - Gene transcription - Glucocorticoid receptor · Obesity · Peroxisome proliferator-activated receptor . Receptor $\cdot$ Rosiglitazone

Abbreviations ADIPOR1: adiponectin receptor 1 . ADIPOR2: adiponectin receptors 2 - Dex: dexamethasone G3PDH: glyceraldehyde-3-phosphate dehydrogenase · GR: glucocorticoid receptor - PEI: polyethylenimine · PMSF: phenylmethylsulfonyl fluoride - PPAR: peroxisome proliferator-activated receptor $\mathrm{RXR}$ : retinoid X receptor . TZDs: thiazolidinediones

\section{Introduction}

Adiponectin is an adipocyte-derived hormone that plays a critical role in the development of type 2 diabetes, which is characterised by hyperglycaemia, insulin resistance and relative impairment of insulin secretion [1,2]. Adiponectin is a $30-\mathrm{kDa}$ protein that contains a short central collagenous region and a C-terminal TNF- $\alpha$-like domain [3-6]. It circulates in the blood at a high concentration (around $10 \mu \mathrm{g} / \mathrm{ml}$ ) as both hexamers and high-molecular-weight oligomers [7]. Adiponectin regulates lipid and glucose metabolism through increases of fatty acid oxidation and glucose uptake, and through a decrease of hepatic gluconeogenesis [1]. In humans, low serum concentrations of adiponectin are associated with insulin resistance, hyperinsulinaemia and an increased risk of type 2 diabetes $[8,9]$. In addition, adiponectin administration is able to decrease the degree of insulin resistance in obese or lipoatrophic mice [10]. The anti-diabetic effect of adiponectin is further supported by the finding that deletion of adiponectin was associated with increased TNF- $\alpha$ concentration in plasma and adipocytes, contributing to severe diet-induced insulin resistance [11].

Adiponectin exerts its biological effect by interacting with adiponectin receptors. Two of these, adiponectin receptors 1 and 2 (ADIPOR1, ADIPOR2), have been cloned and both contain a seven transmembrane motif that 
is different from classical G-protein-coupled receptors in topology [12]. The expression patterns of the two adiponectin receptors are distinct in the embryo and the adult $[12,13]$. ADIPOR1 is ubiquitously expressed with a relatively high level in skeletal muscle. ADIPOR2 is predominantly expressed in the liver. Activation of both ADIPOR 1 and ADIPOR 2 by adiponectin binding is able to stimulate the activities of AMP kinase, a central regulator of intracellular energy metabolism, and such regulation is associated with an increment of fatty acid oxidation and glucose uptake [12]. In addition to ADIPOR1 and ADIPOR2, T-cadherin was recently reported as a receptor for the hexameric and high-molecular-weight species of adiponectin but not for the trimeric or globular species [14].

The peroxisome proliferator-activated receptors (PPARs) belong to a subfamily of nuclear receptors composed of three isoforms, PPARG, PPARA and PPARD [15-17]. PPARs are nutrient sensors that regulate the homeostasis of metabolism. Upon ligand binding, PPARs form heterodimers with retinoid $\mathrm{X}$ receptor (RXR) and then bind to PPAR-responsive elements in the regulatory region of target genes. Thiazolidinediones (TZDs) are a group of PPARG agonists that are widely used in the treatment of type 2 diabetes [18, 19]. Rosiglitazone, a representative TZD, is active in vivo as an anti-diabetic agent in the $o b / o b$ mouse model, and has been used as an oral hypoglycaemic agent in the treatment of type 2 diabetes in humans for many years [20]. The insulin-sensitising activities of PPARG agonists are associated with their ability to decrease the circulating levels of free fatty acids via inhibition of adipocyte lipolysis, as well as regulation of proteins that modulate insulin sensitivity and lipid metabolism [15-17]. Interestingly, PPARG functionally interacts with adiponectin in the regulation of energy metabolism. Adiponectin may mediate the insulin-sensitising effect of TZDs in the liver. The administration of TZDs causes an increase in the serum adiponectin concentrations without affecting body weight in subjects with insulin resistance [21], indicating that adiponectin may function as an early marker of PPARG agonist in the treatment of type 2 diabetes.

To reveal the potential regulation of adiponectin receptors by PPARG agonists in the liver, we analysed the transcriptional regulation of both $A D I P O R 1$ and ADIPOR2. We found that ADIPORI and ADIPOR2 are differently regulated by the PPARG agonist rosiglitazone in hepatocytes, indicating that PPARG may modulate glucose and lipid metabolism through the regulation of adiponectin receptors.

\section{Subjects, materials and methods}

\section{Materials and cell culture}

The human hepatoma cells HepG2 were grown in DMEM supplemented with $10 \%$ heat-inactivated fetal bovine serum, $100 \mu \mathrm{g} / \mathrm{ml}$ streptomycin, and $100 \mathrm{units} / \mathrm{ml}$ penicillin. Rosiglitazone was purchased from Cayman Chemical
(Ann Arbor, MI, USA). Dexamethasone and RU486 were purchased from Sigma (St Louis, MO, USA).

RNA isolation and reverse transcription PCR (RT-PCR)

Total RNA prepared from HepG2 cells or mouse liver was reverse-transcribed with oligo(dT) primer using a SuperScript first-strand synthesis system (Invitrogen, Carlsbad, CA, USA) to generate the first-strand cDNA, followed by PCR to detect the expression of ADIPOR1, ADIPOR 2 and glyceraldehyde-3-phosphate dehydrogenase (G3PDH). The sequences of the PCR primers are as follows: 5'TGCCCTCCTTTCGGGCTTGC-3' and 5'-GCCTTGAC AAAGCCCTCAGCGATAG-3' for human ADIPOR1; 5'GTGTATTCTTCCTGTGCCTGG-3' and 5'-CCACTGGG AGACTATAATGGC-3' for human ADIPOR2; 5'-CAAC GACTACCTGCTACA-3' and 5'-CCCTCAGCGATAG TAAAG-3' for mouse Adipor 1; 5'-TCCTATGCCTTCC TTTCG-3' and 5'-CCAGCC TATCTGCCCTAT-3' for mouse Adipor2. The amplification conditions were as follows: one cycle of denaturing at $94^{\circ} \mathrm{C}$ for $5 \mathrm{~min}$, annealing at $60^{\circ} \mathrm{C}$ for $1 \mathrm{~min}$, and extension at $72^{\circ} \mathrm{C}$ for $1 \mathrm{~min}$. This was followed by 21-31 cycles of PCR. The reaction products were separated on $2 \%$ agarose gel and stained with ethidium bromide.

\section{Western blotting analysis}

HepG2 cells were lysed with $1 \times$ SDS-PAGE loading buffer. The protein was transferred to poly(vinylidene difluoride) (PVDF) membrane that was blocked with 5\% non-fat dry milk in a buffer containing $10 \mathrm{mmol} / \mathrm{l}$ Tris (pH 7.6), $0.9 \% \mathrm{NaCl}$ and $0.1 \%$ Tween-20. The membrane was then probed with an antibody specific for ADIPOR2 (Alpha Diagnostic, San Antonio, TX, USA) or $\alpha$-tubulin (Biomeda, Foster City, CA, USA) followed by anti-mouse HRP (for $\alpha$-tubulin) or anti-rabbit HRP (for ADIPOR2); detection was by enhanced chemiluminescence (ECL; Pierce, Rockford, IL, USA).

Isolation of adiponectin receptor promoters

The $\sim 3.0 \mathrm{~kb}$ human ADIPOR1 promoter and the $\sim 2.0 \mathrm{~kb}$ human $A D I P O R 2$ promoter were cloned by PCR with the human genomic DNA as template and using the primers as follows: 5'-TGCTGTTTCTGGGGCTTCTGAGAGA-3' and 5'-CGGTCCCCCGCGCTACATC-3' for ADIPOR1, and $5^{\prime}$-AGCACACGGTGAACTGTTCCAGAGG-3' and 5'-ACTTCTTGGGAGCCACCGCTGAG-3' for ADIPOR2. The PCR fragments were first cloned into the pBluescript $\mathrm{KS}+$; they were confirmed by DNA sequencing and then subcloned into the pGL3-Basic luciferase vector (Promega, Madison WI, USA). All deletion constructs of the promoter were generated by PCR, followed by subcloning into the pGL3-Basic vector for luciferase assay. 
Luciferase assay

HepG2 cells were seeded in 24-well plates at a density of $2 \times 10^{5}$ cells $/ \mathrm{ml}$. After culturing for $16-24 \mathrm{~h}$, the cells were switched to fresh DMEM without serum and transfected with plasmids using the polyethylenimine (PEI) method [22] with PEI at a molecular weight of 25,000 (Polysciences, Warrington, Philadelphia, PA, USA). A renilla luciferase vector, phRL-SV40 (Promega), was co-transfected to monitor transfection efficiency. Twenty-four hours later, the culture medium was replaced with fresh DMEM containing different drugs. After treatment for $24 \mathrm{~h}$, the cells were harvested by lysis with PBS containing $0.1 \%$ Triton X-100 and $1 \mathrm{mmol} / \mathrm{l}$ phenylmethylsulfonyl fluoride (PMSF). Ten microlitres of the lysate was used in the dualluciferase assay using a method previously described [23]. The samples were counted for $10 \mathrm{~s}$ with a luminometer (Berthold, Bad Wildbad, Germany). The luciferase activity was normalised to the renilla luciferase activity and triplicate transfections were carried out for each treatment. At least three separate transfection experiments were performed for each assay. Student's $t$-test was used to analyse the luciferase data by comparing the untreated and treated samples.

Animal studies

Five-week-old C57BL/6 mice (SLAC, Shanghai, China) were housed two or three mice per cage and allowed free access to standard rodent chow and water. The animals were dosed daily by gavage with either PBS (as control) or rosiglitazone (Avandia, GlaxoSmithKline, Brentford, Middlesex, UK) at $30 \mathrm{mg} \cdot \mathrm{kg}^{-1} \cdot \mathrm{day}^{-1}$. Mice were killed after 10 days of treatment and the livers were dissected and used for the experiment.

\section{Results}

PPARG agonist is able to elevate the ADIPOR2 mRNA and protein levels in HepG2 cells

First, the effect of the PPARG agonist on the regulation of adiponectin receptors was analysed in a human hepatoma cell line, HepG2 cells [24]. HepG2 cells were treated with either DMSO or the PPARG agonist rosiglitazone. Total RNA was isolated from the cells and used in RT-PCR to analyse the levels of ADIPOR1 and ADIPOR2 mRNA. As shown in Fig. 1a, we were able to detect the expression of both $A D I P O R 1$ and ADIPOR 2 in HepG 2 cells. Treatment of HepG2 cells with rosiglitazone did not significantly influence the level of ADIPOR 1 mRNA. However, rosiglitazone was able to strongly stimulate $A D I P O R 2$ expression. To monitor the relative RNA amounts used in the experiment, the same RNA samples were also used in PCR with primers specific for the housekeeping gene, $G 3 P D H$. As shown in Fig. 1a, the G3PDH levels were consistent among the samples. In addition, the protein level of ADIPOR2 was analysed in the HepG2 cells using a specific antibody. As shown in Fig. 1b, rosiglitazone treatment in HepG2 cells was also able to elevate the protein level of ADIPOR2 in a dose-dependent manner, but not the level of the loading control $\alpha$-tubulin. Therefore, these data provided the first evidence that rosiglitazone is able to selectively upregulate $A D I P O R 2$ expression in HepG2 cells.

\section{Regulation of ADIPOR2 promoter by rosiglitazone}

To further analyse the regulation of adiponectin receptors by PPARG agonist at the transcriptional level, we isolated the 5' regulatory regions of both ADIPOR1 and ADIPOR2, including $\mathrm{a} \sim 3 \mathrm{~kb}$ human $A D I P O R \mathrm{l}$ promoter region $(-3,133$ to -125 bp relative to the translation initiation site) and $\mathrm{a} \sim 2 \mathrm{~kb}$ human $A D I P O R 2$ promoter region $(-2,171$ to $-99 \mathrm{bp}$ relative to the translation initiation site). Both of the putative adiponectin promoters were linked to a luciferase reporter and transiently transfected into HepG2 cells.

\section{a}
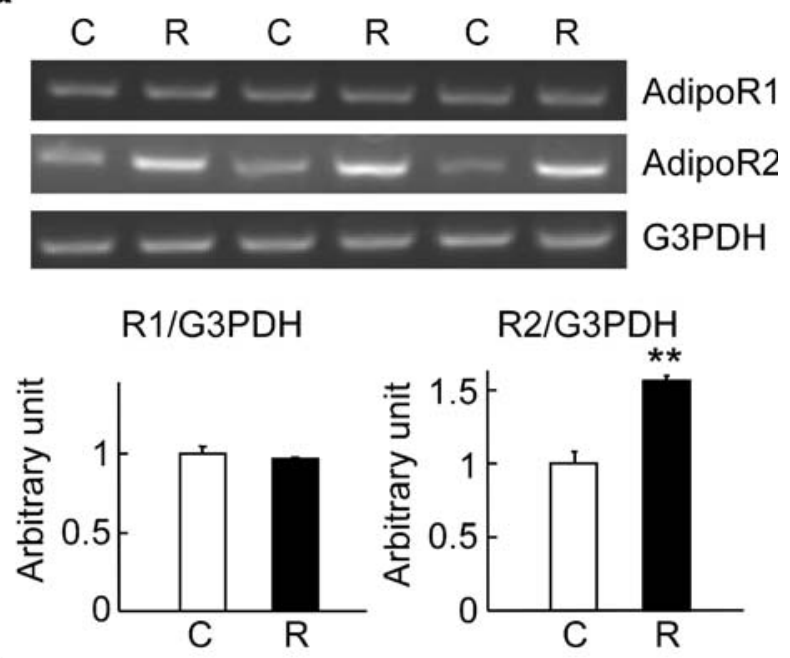

b

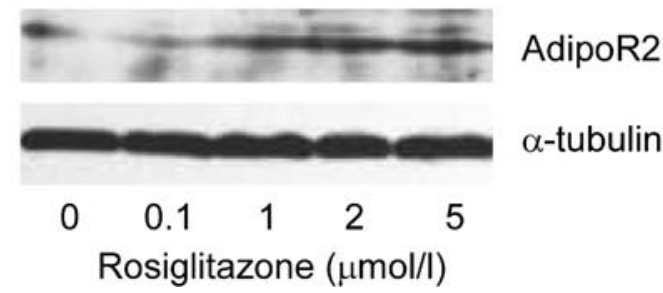

Fig. 1 Effect of the PPARG agonist rosiglitazone on the expression of adiponectin receptors in HepG2 cells. a The mRNA level for adiponectin receptors. HepG2 cells were treated with DMSO (control; C) or $100 \mathrm{nmol} / 1$ rosiglitazone $(R)$ for $24 \mathrm{~h}$ and the total RNA was used for RT-PCR with specific primers for ADIPOR1, ADIPOR2 and G3PDH. The relative values of adiponectin receptors for each sample were compared with those of G3PDH and shown as average $\pm \mathrm{SD}$. Student's $t$-test was performed on the data; $* * p<0.01$. b The protein levels of adiponectin receptors. The rosiglitazonetreated HepG2 cells were used in western blotting analysis with antibodies for either ADIPOR2 or $\alpha$-tubulin (as control) 
a

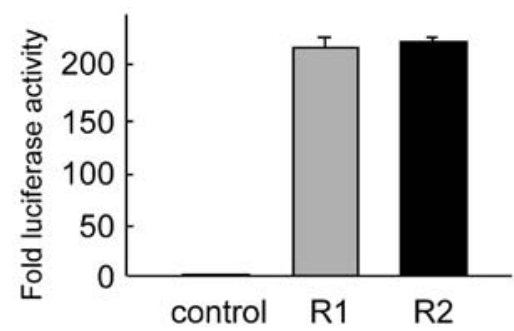

b

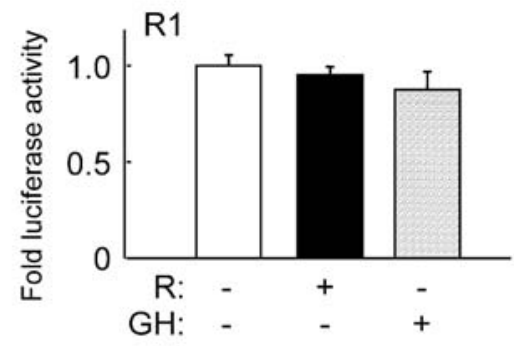

C

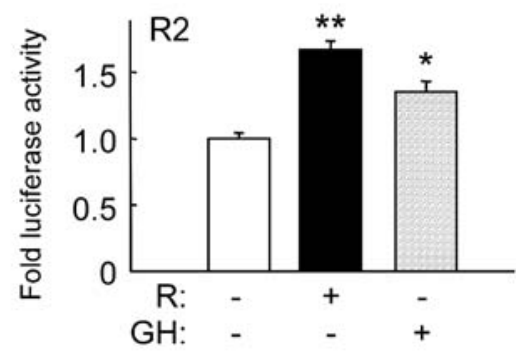

d

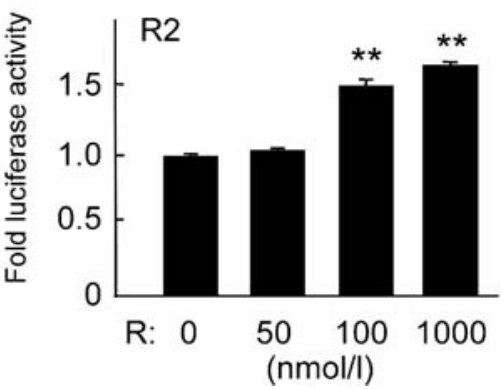

Fig. 2 Regulation of $A D I P O R 1$ and $A D I P O R 2$ promoters. a The luciferase vector control or the human ADIPORl $(R 1)$ and $A D I P O R 2(R 2)$ promoter luciferase constructs were transiently transfected into HepG2 cells. Renilla luciferase vector was used to monitor the transfection efficiency. The whole-cell lysate was used in the dual-luciferase assay $48 \mathrm{~h}$ after transfection. The fold change of luciferase activity is shown as mean \pm SD. b The effect of rosiglitazone $(R)$ and growth hormone $(G H)$ on ADIPOR1 promoterluciferase reporter. HepG2 cells were transfected with the $A D I$ POR1-luciferase construct and treated with $100 \mathrm{nmol} / 1$ rosiglitazone or $500 \mathrm{ng} / \mathrm{ml} \mathrm{GH}$ for $24 \mathrm{~h}$ before luciferase assay. c The same experiment as in (b) was performed with the ADIPOR2 luciferase plasmid. d Effect of different concentrations of rosiglitazone on ADIPOR2 promoter activity. Student's $t$-test was performed on the data; ${ }^{*} p<0.05 ; * * p<0.01$

Meanwhile, a renilla luciferase vector driven by SV40 promoter was co-transfected to monitor the transfection efficiency. As shown in Fig. 2a, both promoters exhibited very high basal activities in HepG2 cells when compared with the empty luciferase vector, indicating that the $5^{\prime}$ regulatory regions of $A D I P O R 1$ and $A D I P O R 2$ did contain regulatory elements to drive the expression of both genes in HepG2 cells. When the luciferase construct of the ADIPOR1 promoter was transfected into HepG2 cells, neither rosiglitazone nor growth hormone was able to activate the promoter activity (Fig. 2b). However, both rosiglitazone and growth hormone were able to elevate ADIPOR2 promoter activity significantly (Fig. 2c), indicating that the transcription of ADIPOR2 was positively regulated by the PPARG agonist as well as by growth hormone. The effect of growth hormone on ADIPOR2 regulation was also consistent with the observation in 3T3L1 adipocytes [25]. Furthermore, we found that rosiglitazone was able to increase the transactivation of ADIPOR2 promoter in a dose-dependent fashion. As shown in Fig. 2d, the stimulation of ADIPOR 2 promoter by rosiglitazone increased with increasing dose of rosiglitazone. Taken together, these data suggest that the ADIPOR2 promoter is positively regulated by PPARG activator in HepG2 cells.

Identification of a rosiglitazone-responsive region in the ADIPOR2 promoter

In an attempt to identify the region of the human ADIPOR2 promoter responsive to the stimulatory effect of rosiglitazone, we generated a series of deletion constructs of the ADIPOR2 promoter and fused them to the luciferase reporter. Those deletion constructs were then transfected into HepG2 cells for the luciferase assay. As shown in Fig. 3, rosiglitazone treatment was significantly stimulated the promoter $(\mathrm{R} 2$, the $\sim 2 \mathrm{~kb} A D I P O R 2$ promoter; and the $\Delta \mathrm{A}$ to $\triangle \mathrm{E}$ constructs) until it was shortened to $-401 \mathrm{bp}$ upstream of the translation initiation site $(\Delta \mathrm{F}$ construct). A further deletion of the promoter also led to loss of the stimulatory effect of rosiglitazone ( $\Delta \mathrm{G}$ construct). These data suggest that the promoter sequences between $-451 \mathrm{bp}$ (the $\Delta \mathrm{E}$ construct) and $-401 \mathrm{bp}$ (the $\Delta \mathrm{F}$ construct) contain a critical regulatory site(s) that enables the promoter to be responsive to rosiglitazone in HepG2 cells.

To further characterise the rosiglitazone-responsive region of the human $A D I P O R 2$ promoter, we analysed a few more deletion mutants of the promoter, from -451 to $-431 \mathrm{bp}$ relative to the translation initiation site. As shown in Fig. $4 \mathrm{a}$, we found that both the $-451 \mathrm{bp}(\Delta \mathrm{E})$ and -446 bp $(\Delta \mathrm{H})$ constructs were responsive to rosiglitazone stimulation. However, the deletion constructs $-441 \mathrm{bp}$ $(\Delta \mathrm{I}),-436 \mathrm{bp}(\Delta \mathrm{J})$ and $-431 \mathrm{bp}(\Delta \mathrm{K})$ lost the ability to mediate rosiglitazone activation of the promoter (Fig. 4a). Taken together, these results indicated that the sequence between -446 and $-441 \mathrm{bp}$ was critical for the stimulatory activity of rosiglitazone. 


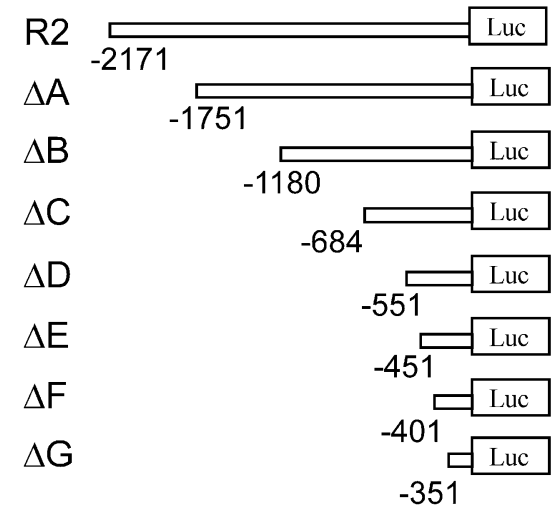

. 3 Characterisation of the human ADIPOR2 promoter. The lefthand side of the figure illustrates the lengths of the different promoter constructs with the number indicating the distance of the constructs in base pairs relative to the translation initiation site. HepG2 cells were transiently transfected with the various deletion constructs of the ADIPOR2 promoter as indicated and co-transfected

Functional involvement of a glucocorticoidreceptor-binding site in the ADIPOR2 promoter

After the characterisation of the putative rosiglitazoneresponsive region of the $A D I P O R 2$ promoter, we analysed

\section{a}

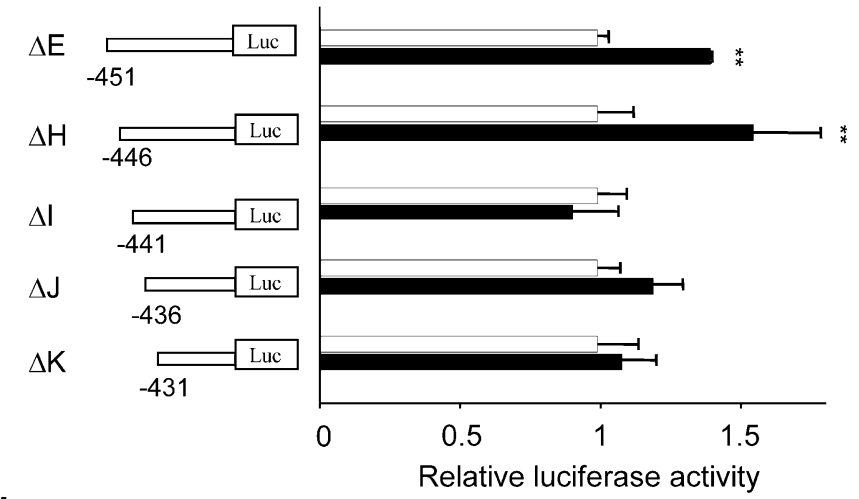

b

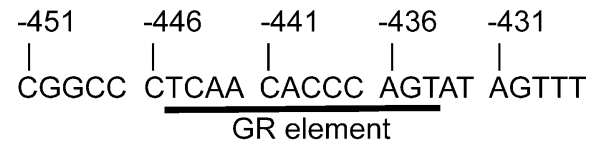

Fig. 4 Identification of a rosiglitazone-responsive region on the ADIPOR2 promoter. a Luciferase assay with different deletion constructs. The left-hand side depicts the length of the promoter constructs with the number indicating the distance of the constructs relative to the translation initiation site. HepG2 cells were transfected with various deletion constructs as indicated. Twenty-four hours after transfection, the cells were treated with DMSO as control (blank bars) or $100 \mathrm{nmol} / 1$ rosiglitazone (filled bars) for $24 \mathrm{~h}$ followed by dual luciferase assay. The fold change of luciferase activity is shown as mean $\pm \mathrm{SD}$. Student's $t$-test was performed with the data; ${ }^{* *} p<0.01$. b The nucleotide sequence contained in these deletion constructs of the promoter; the putative glucocorticoid receptor element is indicated

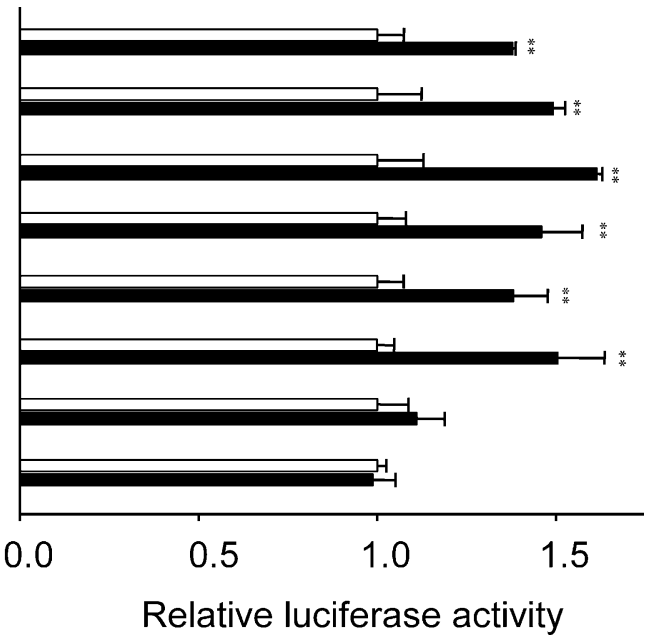

1307

with renilla luciferase vector. Twenty-four hours after transfection, the cells were treated with DMSO as control (blank bars) or $100 \mathrm{nmol} / 1$ rosiglitazone (filled bars) for $24 \mathrm{~h}$ followed by dual luciferase assay. The fold change of luciferase activity is shown as mean \pm SD. Student's $t$-test was performed; $* * p<0.01$

a

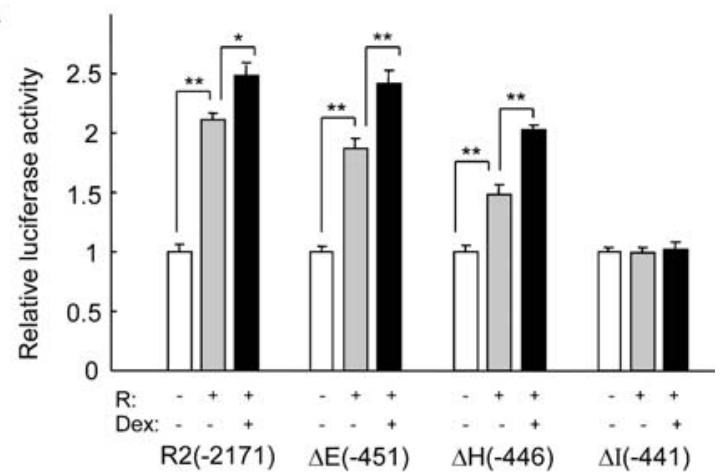

b

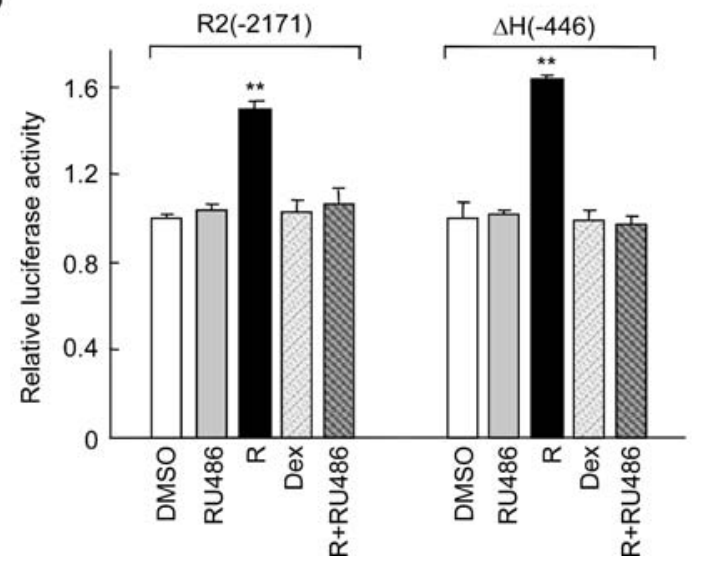

Fig. 5 Effects of GR agonist and antagonist on the ADIPOR2 promoter. a HepG2 cells were transiently transfected with various ADIPOR2 promoter constructs as indicated with the number indicating the length of the constructs relative to the translation initiation site. The cells were then treated with $100 \mathrm{nmol} / \mathrm{l}$ rosiglitazone $(R)$ with or without $10 \mathrm{nmol} / 1$ dexamethasone $(D e x)$ for $24 \mathrm{~h}$. The data shown here are representative of three independent experiments as fold change $\pm \mathrm{SD}$. Student's $t$-test was performed with the data; ${ }^{*} p<0.05$; $* * p<0.01$. b A similar experiment was performed with treatment of the cells with RU486 $(1 \mu \mathrm{mol} / \mathrm{l})$ and other drugs as indicated 

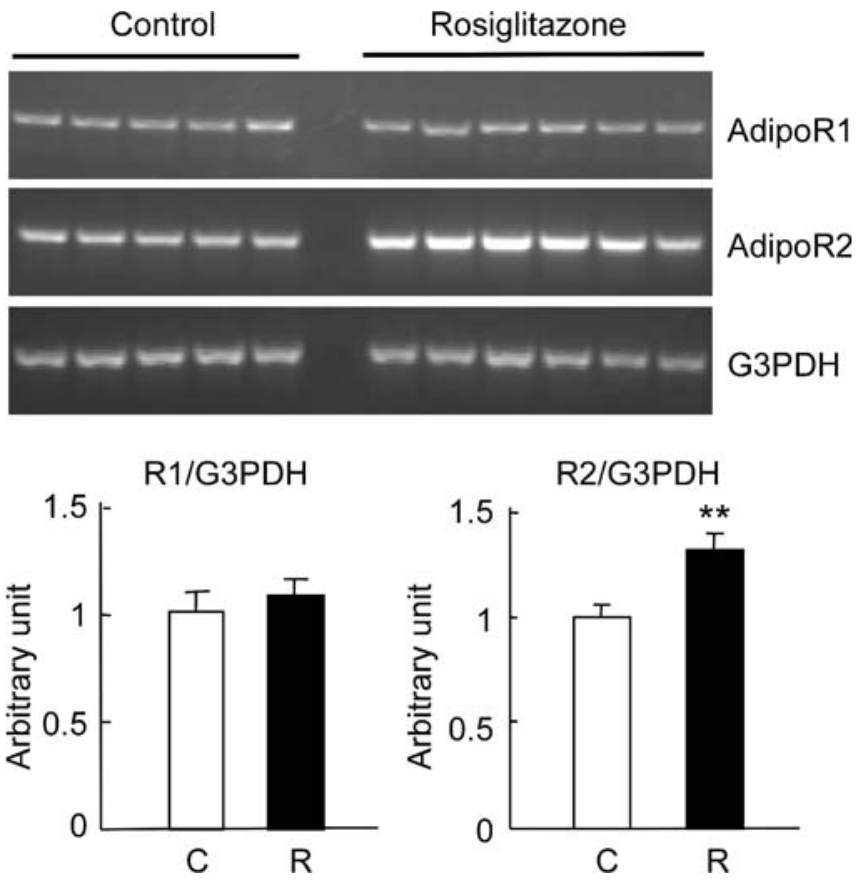

Fig. 6 Effect of rosiglitazone on the expression of adiponectin receptors in mouse liver. C57BL/6 mice were treated with PBS control ( $n=5$, three females, two males) or rosiglitazone $(n=6$, three females, three males) for 10 days. Mouse livers were used in RTPCR to determine the mRNA levels of ADIPOR1, ADIPOR2 and G3PDH. The PCR results were scanned (a) and the intensity of each band was quantified. The relative value of ADIPOR1 (b) and ADIPOR2 (c) for each sample was compared with that of G3PDH and shown as mean $\pm \mathrm{SD}$. $C$, control; $R$, rosiglitazone treatment. Student's $t$-test was performed; ${ }^{* *} p<0.01$

the enclosed nucleotide sequence in this responsive region to determine the putative transcription factor binding sites using the TESS program (provided by the Computational Biology and Informatics Laboratory at the University of Pennsylvania Schools of Medicine Engineering and Applied Science at (http://www.cbil.upenn.edu). As a result, we found a consensus glucocorticoid receptor (GR) binding element in this region (Fig. 4b), instead of a classical PPAR-responsive element [15-17]. It is significant that the human ADIPOR1 promoter did not contain such a GR element in the corresponding region. It has previously been reported that the PPARG agonist has an effect on GR-mediated gene transcription through interaction between PPARG and GR [26]. Therefore, we hypothesised that such an interaction might be implicated in the stimulatory effect of rosiglitazone on the ADIPOR2 receptor. To address this hypothesis, we investigated the combined effect of rosiglitazone and dexamethasone (Dex, a ligand for GR) on the transactivation of the ADIPOR2 promoter. As shown in Fig. 5a, rosiglitazone treatment stimulated various $A D I P O R 2$ promoter constructs (R2, the $\sim 2 \mathrm{~kb}$ promoter; $\Delta \mathrm{E}$ and $\Delta \mathrm{H}$ deletion constructs). Interestingly, treatment with Dex was able to activate the promoter activity with these constructs further, indicating a synergistic or additive effect between rosiglitazone and Dex. As expected, the $\Delta \mathrm{I}$ promoter construct that lost the putative GR site was no longer responsive to the stimulatory effect of rosiglitazone or to the synergistic effect of Dex. We also analysed the effect of a GR antagonist RU486 on the stimulation of ADIPOR2 transcription by rosiglitazone. As shown in Fig. 5b, RU486 or Dex alone had no obvious effect on the promoter activities of either full-length or $\Delta \mathrm{H}$ promoter constructs. However, RU486 was able to abrogate the stimulatory effect of rosiglitazone on the ADIPOR 2 promoter. Taken together, these data suggest that rosiglitazone stimulation on ADIPOR 2 transcription is probably mediated by the GR element located in the rosiglitazone-responsive region of the promoter.

Rosiglitazone is able to stimulate ADIPOR2 expression in mouse liver

We also investigated the in vivo effect of the PPARG agonist on ADIPOR2 expression in the liver. C57BL/6 mice were randomly divided into two groups and were treated with either PBS or rosiglitazone for 10 days. The livers of the animals were used in RT-PCR to determine the levels of mRNA for ADIPOR1 and ADIPOR2 in comparison with that of the housekeeping gene $G 3 P D H$. As shown in Fig. 6, rosiglitazone treatment had no effect on the level of ADIPOR1 mRNA. However, it was able to significantly elevate the expression of ADIPOR2. Therefore, these experiments provided additional evidence that the PPARG agonist is able to positively regulate $A D I P O R 2$ expression in liver cells.

\section{Discussion}

In this study, we analysed the transcriptional regulation of adiponectin receptors ADIPOR1 and ADIPOR2 in HepG2 hepatoma cells and mouse livers. We found that the PPARG agonist rosiglitazone was able to elevate both the mRNA and protein levels, as well as to stimulate the promoter activity of $A D I P O R 2$ but not ADIPOR1 in HepG2 cells. In addition, treatment of mice with rosiglitazone stimulated ADIPOR 2 expression in the liver. Detailed molecular analyses demonstrated a putative rosiglitazone-responsive region in the $A D I P O R 2$ promoter. Interestingly, this rosiglitazone-responsive region does not contain a classical consensus PPAR-binding sequence. Instead, it is composed of a GR-binding element. Consistently, the GR agonist dexamethasone was able to synergise with rosiglitazone to stimulate the ADIPOR2 promoter. Therefore, these studies suggest that the PPARG agonist is able to functionally interact with the GR element in the ADIPOR2 promoter to mediate the stimulatory activity of rosiglitazone on gene transcription.

Our study indicates a new therapeutic action of PPARG in the amelioration of insulin resistance in type 2 diabetes. It has been reported that administration of TZDs increased serum adiponectin concentrations without affecting body weight in subjects with insulin resistance [21]. Our studies demonstrated that the PPARG agonist causes an increase in 
ADIPOR2 transcription in liver cells and this effect is expected to enhance the biological activities of adiponectin in the liver. Therefore, the stimulation of both adiponectin and $A D I P O R 2$ expression by TZDs may have a synergistic effect on the modulation of glucose and lipid metabolism by these drugs in the treatment of type 2 diabetes.

In humans, the expression and regulation of adiponectin receptors are associated with type 2 diabetes. In a study of a Mexican-American population, the expression levels of ADIPOR1 and ADIPOR2 in skeletal muscles and the plasma adiponectin concentration were lower in people with a family history of type 2 diabetes than in those without the family history [27]. This is consistent with the fact that the expression levels of both adiponectin receptors in human skeletal muscle cells were related to distinct in vivo parameters of glucose and lipid metabolism [28]. Genetic studies in an Old-Order Amish population also revealed that genetic variations in both adiponectin receptors, including the $5^{\prime}$ promoter regions, are associated with type 2 diabetes [29]. In addition, rosiglitazone treatment of type 2 diabetes patients is associated with an increase of ADIPOR1 level in adipose tissues and a decrease of ADIPOR1 in skeletal muscle [30]. In $o b / o b$ mice, the expression of both ADIPOR1 and ADIPOR2 is decreased in skeletal muscle and adipose tissues compared to control animals; this decrease is via insulin-mediated repression of the receptor expression through the PI3kinase/Foxol pathway [31]. At the cellular level, it has been reported that growth hormone is able to stimulate the level of $A D I P O R 2$ mRNA but not that of ADIPOR1 mRNA in 3T3-L1 adipocytes [25]. Our studies found that growth hormone was also able to slightly increase the transcription of $A D I P O R 2$, but not $A D I P O R 1$, in liver cells (Fig. 2); this further supports the regulatory role of growth hormone on the expression of adiponectin receptors in different cell types. Consistent with our observation, the PPARG agonist has been shown to upregulate the level of ADIPOR2 mRNA in macrophages [32]. Therefore, both these studies and our findings indicate that the expression of adiponectin receptors is regulated by different pathways in different cell types and that regulation is likely to be associated with the altered glucose and lipid metabolism in either type 2 diabetes or after treatment with anti-diabetic drugs.

It is noteworthy that the stimulatory effect of PPARG agonist on $A D I P O R 2$ transcription that was observed is not directly through a classical PPAR-binding element in the promoter. Our serial deletion studies with the ADIPOR 2 promoter indicated that rosiglitazone activates ADIPOR2 promoter through a conserved GR sequence. Deletion of the consensus GR sequence was able to abrogate the stimulatory effect of rosiglitazone on ADIPOR2 promoter. In addition, the GR agonist dexamethasone appeared to have an additive/synergistic effect with rosiglitazone on the transactivation of ADIPOR 2 promoter. Furthermore, the GR antagonist RU486 was able to cancel the stimulatory effect of rosiglitazone on the ADIPOR2 promoter. Collectively, these data indicate a functional involvement of the GR element in the regulation of ADIPOR2 in liver cells. It has previously been reported that the PPARG agonist $15 \mathrm{~d}-\mathrm{PGJ}_{2}$ is able to induce protein-protein interactions between PPARG and GR in TNF- $\alpha$-treated human airway smooth muscle cells and such interactions are implicated in PPARG inhibition of the TNF- $\alpha$ mediated production of eotaxin and monocyte chemotactic protein-1 [26]. In liver cells, rosiglitazone may induce a similar interaction between PPARG and GR, leading to transcriptional upregulation of ADIPOR2 expression. This is further supported by a recent report showing that the anti-inflammatory activity of the PPARG agonists rosiglitazone and ciglitazone is reversed by RU486 [33]. However, the detailed molecular mechanism underlying the regulation of $A D I P O R 2$ transcription through functional interaction between PPARG and GR remains to be elucidated.

In conclusion, this is the first report that demonstrates the stimulatory effect of a PPARG agonist on the transcriptional regulation of $A D I P O R 2$ in liver cells. It reveals a new paradigm that underlies the therapeutic effect of PPARG activators in the treatment of type 2 diabetes. The increased expression of $A D I P O R 2$ is expected to lead to an increased sensitivity of liver cells to adiponectin and thereby to enhancement of the anti-diabetic activity of adiponectin.

Acknowledgements This work was supported by research grants from the Chinese Academy of Sciences (Bairen Plan), the National Natural Science Foundation of China (30588002), and the Science \& Technology Commission of Shanghai Municipality (04dz14007) to Y. Chen.

Duality of Interest The authors declare that they have no duality of interest.

\section{References}

1. Kadowaki T, Yamauchi T (2005) Adiponectin and adiponectin receptors. Endocr Rev 26:439-451

2. Trujillo ME, Scherer PE (2005) Adiponectin-journey from an adipocyte secretory protein to biomarker of the metabolic syndrome. J Intern Med 257:167-175

3. Scherer PE, Williams S, Fogliano M, Baldini G, Lodish HF (1995) A novel serum protein similar to $\mathrm{Clq}$, produced exclusively in adipocytes. J Biol Chem 270:26746-26749

4. Hu E, Liang P, Spiegelman BM (1996) AdipoQ is a novel adipose-specific gene dysregulated in obesity. J Biol Chem 271:10697-10703

5. Maeda K, Okubo K, Shimomura I, Funahashi T, Matsuzawa Y, Matsubara K (1996) cDNA cloning and expression of a novel adipose specific collagen-like factor, apM1 (AdiPose Most abundant Gene transcript 1). Biochem Biophys Res Commun 221:286-289

6. Nakano Y, Tobe T, Choi-Miura NH, Mazda T, Tomita M (1996) Isolation and characterization of GBP28, a novel gelatinbinding protein purified from human plasma. J Biochem (Tokyo) 120:803-812

7. Waki H, Yamauchi T, Kamon $J$ et al (2003) Impaired multimerization of human adiponectin mutants associated with diabetes. Molecular structure and multimer formation of adiponectin. J Biol Chem 278:40352-40363

8. Lindsay RS, Funahashi T, Hanson RL et al (2002) Adiponectin and development of type 2 diabetes in the Pima Indian population. Lancet 360:57-58 
9. Weyer C, Funahashi T, Tanaka S et al (2001) Hypoadiponectinemia in obesity and type 2 diabetes: close association with insulin resistance and hyperinsulinemia. J Clin Endocrinol Metab 86:1930-1935

10. Yamauchi T, Kamon J, Waki $\mathrm{H}$ et al (2001) The fat-derived hormone adiponectin reverses insulin resistance associated with both lipoatrophy and obesity. Nat Med 7:941-946

11. Maeda N, Shimomura I, Kishida K et al (2002) Diet-induced insulin resistance in mice lacking adiponectin/ACRP30. Nat Med 8:731-737

12. Yamauchi T, Kamon J, Ito $\mathrm{Y}$ et al (2003) Cloning of adiponectin receptors that mediate antidiabetic metabolic effects. Nature 423:762-769

13. Zhou Y, Sun X, Jin L, Stringfield T, Lin L, Chen Y (2005) Expression profiles of adiponectin receptors in mouse embryos. Gene Expr Patterns 5:711-715

14. Hug C, Wang J, Ahmad NS, Bogan JS, Tsao TS, Lodish HF (2004) T-cadherin is a receptor for hexameric and highmolecular-weight forms of Acrp30/adiponectin. Proc Natl Acad Sci USA 101:10308-10313

15. Desvergne B, Michalik L, Wahli W (2004) Be fit or be sick: peroxisome proliferator-activated receptors are down the road. Mol Endocrinol 18:1321-1332

16. Berger J, Moller DE (2002) The mechanisms of action of PPARs. Annu Rev Med 53:409-435

17. Li AC, Glass CK (2004) PPAR- and LXR-dependent pathways controlling lipid metabolism and the development of atherosclerosis. J Lipid Res 45:2161-2173

18. Nolan JJ, Ludvik B, Beerdsen P, Joyce M, Olefsky J (1994) Improvement in glucose tolerance and insulin resistance in obese subjects treated with troglitazone. $\mathrm{N}$ Engl J Med 331:1188-1193

19. Iwamoto Y, Kosaka K, Kuzuya T, Akanuma Y, Shigeta Y, Kaneko T (1996) Effects of troglitazone: a new hypoglycemic agent in patients with NIDDM poorly controlled by diet therapy. Diabetes Care 19:151-156

20. Mayerson AB, Hundal RS, Dufour S et al (2002) The effects of rosiglitazone on insulin sensitivity, lipolysis, and hepatic and skeletal muscle triglyceride content in patients with type 2 diabetes. Diabetes 51:797-802

21. Maeda N, Takahashi M, Funahashi T et al (2001) PPARgamma ligands increase expression and plasma concentrations of adiponectin, an adipose-derived protein. Diabetes 50:2094-2099
22. Godbey WT, Wu KK, Hirasaki GJ, Mikos AG (1999) Improved packing of poly(ethylenimine)/DNA complexes increases transfection efficiency. Gene Ther 6:1380-1388

23. Nagarajan RP, Zhang J, Li W, Chen Y (1999) Regulation of smad7 promoter by direct association with smad3 and smad4. J Biol Chem 274:33412-33418

24. Knowles BB, Howe CC, Aden DP (1980) Human hepatocellular carcinoma cell lines secrete the major plasma proteins and hepatitis B surface antigen. Science 209:497-499

25. Fasshauer M, Klein J, Kralisch S et al (2004) Growth hormone is a positive regulator of adiponectin receptor 2 in 3T3-L1 adipocytes. FEBS Lett 558:27-32

26. Nie M, Corbett L, Knox AJ, Pang L (2005) Differential regulation of chemokine expression by peroxisome proliferatoractivated receptor gamma agonists: interactions with glucocorticoids and beta2-agonists. J Biol Chem 280:2550-2561

27. Civitarese AE, Jenkinson CP, Richardson D et al (2004) Adiponectin receptors gene expression and insulin sensitivity in non-diabetic Mexican Americans with or without a family history of Type 2 diabetes. Diabetologia 47:816-820

28. Staiger H, Kaltenbach S, Staiger K et al (2004) Expression of adiponectin receptor mRNA in human skeletal muscle cells is related to in vivo parameters of glucose and lipid metabolism. Diabetes 53:2195-2201

29. Damcott CM, Ott SH, Pollin TI et al (2005) Genetic variation in adiponectin receptor 1 and adiponectin receptor 2 is associated with type 2 diabetes in the Old Order Amish. Diabetes $54: 2245-2250$

30. Tan GD, Debard C, Funahashi T et al (2005) Changes in adiponectin receptor expression in muscle and adipose tissue of type 2 diabetic patients during rosiglitazone therapy. Diabetologia 48:1585-1589

31. Tsuchida A, Yamauchi T, Ito $Y$ et al (2004) Insulin/Foxo1 pathway regulates expression levels of adiponectin receptors and adiponectin sensitivity. J Biol Chem 279:30817-30822

32. Chinetti G, Zawadski C, Fruchart JC, Staels B (2004) Expression of adiponectin receptors in human macrophages and regulation by agonists of the nuclear receptors PPARalpha, PPARgamma, and LXR. Biochem Biophys Res Commun 314:151-158

33. Ialenti A, Grassia G, Di Meglio P, Maffia P, Di Rosa M, Ianaro A (2005) Mechanism of the anti-inflammatory effect of thiazolidinediones: relationship with the glucocorticoid pathway. Mol Pharmacol 67:1620-1628 